\title{
Recall as a self-limiting process
}

\author{
HENRY L. ROEDIGER III \\ Purdue University, West Lafayette, Indiana 47907
}

\begin{abstract}
Presentation of only some category names as retrieval cues following presentation of a categorized list enhanced recall of the cued categories relative to free recall, but reduced recall of the noncued categories (Experiment 1). This recall interference from recall of cued categories increased with the number of category names presented (Experiment 2), and was not due merely to delaying the subject with an interfering interpolated task (Experiment 3). The results argue against theories assuming that categories are recalled via associations from previously recalled categories. Rather, the results suggest that recall of categories acts in a manner to limit recall of additional categories, in agreement with the characterization of recall as a sampling-withreplacement process as described by Rundus.
\end{abstract}

The central importance of retrieval processes in human memory has been widely recognized since publication of Tulving and Pearlstone's (1966) landmark experiment. Briefly, they showed that the amount of information recalled depends critically on the nature of retrieval cues embedded in the test situation. When conditions affecting storage of material are held constant, recall can still be greatly affected by varying the conditions under which the information is retrieved. Characterizing the nature of the retrieval process underlying recall thus becomes a primary task for psychologists interested in memory.

The retrieval process underlying recall is usually thought of as self-propagating: Recall of some information aids and guides recall of other information later. This self-propagating idea of recall is embedded in all associative and organizational theories of recall. For example, Anderson's (1972) model for free recall assumes that to-be-remembered information, such as words in a list, are associated with each other and then at recall the retrieval process is sustained by these interitem associations. Recall of items early in the recall period allows additional items to be recalled through the mechanism of associative chaining. Recall is self-propagating because the act of recall itself provides the cues that guide later recall. Such a proposal was originally made by Aristotle and the intervening 2,000 years have done little to damage the general idea.

The purpose of the present paper is to present evidence that recall not only facilitates and guides later recall, but also limits the amount of additional information that can be recalled. The very act of recall seems to attenuate recall of additional information. Thus recall may be considered a self-limiting process.

This article was written while the author was on leave at the University of Toronto. I would like to thank John M. Gardiner, Norman J. Slamecka, and Endel Tulving for commenting on an earlier version of the manuscript. Requests for reprints may be sent to Henry L. Roediger, III, Department of Psychology, Purdue University, West Lafayette, Indiana 47907.
The idea that recall is a source of forgetting is not new and is usually referred to as output interference. Before presenting evidence from a new paradigm, previous work will be briefly reviewed.

The first systematic studies of output interference were performed by Tulving and Arbuckle (1963), who presented subjects with 10 paired associates composed of single digits and words. At recall the subjects were cued with the digits in a counterbalanced order and recall of words was examined as a function of output position. Recall declined as a function of output position only for the last few items presented in the list, which has led more recently to the conclusion that items held in primary memory are subject to output interference but that those held in secondary memory are not. Just as other distractor tasks can eliminate recall from primary memory, so can the act of recall itself. By this view output interference is no more than a nonspecific source of interference, no different (and no more interesting) than mental arithmetic or other distractor tasks employed in the study of short-term memory.

The finding that the act of recall damages items held in primary memory has been complemented by the results of other experiments showing output interference in secondary memory (e.g., Dong, 1972; Smith, 1971; Smith, D'Agostino, \& Reid, 1970). Subjects were presented with blocked categorized word lists and at recall subjects were given the category names in a counterbalanced order and asked to recall as many items as possible from each category. The typical finding from these studies is that recall of words within the categories decreases systematically with output position of the category. This cannot be attributed only to recall of the last few items, because the effect is independent of input order of the categories. Also, the effect is obtained when an interpolated task is provided between presentation and recall or when the last category presented is omitted from the results (Smith, 1971). Thus, the evidence is quite convincing 
for an effect of output interference or recall interference in secondary memory.

A second phenomenon analogous to output interference is the deleterious effect of presenting items from a list as cues for other items (Slamecka, $1968,1969)$. When subjects are presented with some items from a list as cues for the remaining list items. they actually recall fewer of these remaining target items than other subjects engaged in free recall but scored on the same set of target items. Furthermore, the proportion of the target items recalled decreases with increases in the number of items presented as cues (Roediger, 1973; Rundus, 1973; Watkins, 1975). It has been argued that study of part-list cues is analogous to recall of the same items and that the consequent inhibiting effect on recall of other items is similar to recall interference (Roediger, 1974). Crowder (1976, pp. 342-351) provides a review of research in this area.

The present experiments were designed to explore a third variety of recall interference in categorized list recall. These experiments are analogous to those where list cues were presented, but in these experiments subjects were given some category names from a list and the effect of these cues on recall of words in other categories was observed. Subjects heard blocked categorized lists of eight categories with instances from a category preceded by the appropriate category name. After performing a distractor task, different groups of subjects in Experiment 1 were instructed to recall the list in different ways. One group of subjects was given free recall instructions, while a second group was given similar instructions but provided with all eight category names as cues. The other two groups were of primary interest. One group received four category names at recall, with instructions to forget or ignore words in those categories and only to recall words in the four categories that were not named. The final group of subjects was also provided four category names, but was told to recall as many words as possible from the entire list, both from the cued and noncued categories. The primary interest centers on the recall of words from the categories in the last two conditions, whose names did not appear on the recall sheets, relative to free recall of the same words. By the view that additional categories are recalled by associative chaining from categories activated earlier (e.g., Anderson, 1972), provision of category names should aid recall of additional categories by providing associative paths that would otherwise have been unavailable. The benefit to recall of noncued categories would presumably occur whether or not subjects were asked to recall words from the categories whose names were presented as cues.

On the other hand, if there are no direct associative links between category representations, as is assumed in a theory of recall proposed by Rundus (1973), the presentation of category names for some categories would not be expected to aid recall of words in other categories. In fact, according to Rundus' (1973) theory, one would expect that presentation of some category names should actually reduce recall of words from the other, noncued, categories relative to free recall. Briefly, this is because Rundus (1973) assumes that recall serves to strengthen the representation of information in menory and that on future retrieval attempts this previously recalled information competes with retrieval of new information. (Rundus' model is discussed more fully in the General Discussion.) Thus, in the first experiment, presentation of four category names should serve to reduce recall of words from the remaining four categories relative to free recall because the cued (and strengthened) categories should compete with recall of noncued categories. This reduction should be greater in the case where subjects are asked to recall the words from the category than when they are told that they need not recall the words, because the act of recalling the words in the category should serve to strengthen the category representation more greatly than simply presenting the category names. An experiment based on similar logic has been reported by Parker and Warren (1974) and is discussed with the results from Experiment 1.

\section{EXPERIMENT 1}

\section{Method}

Subjects. The subjects were 96 Purdue University undergraduates who participated to fulfill a course requirement. They were tested in groups of 10 to 20 .

Materials. Two categorized lists were constructed that contained eight categories and eight items per category. Consequently, each list contained 64 words. The 16 categories used in the experiment were taken from the Battig and Montague (1969) category norms. The items selected from each category were of medium frequency to minimize chance hits due to guessing.

Design. Subjects recalled the two lists under one of four experimental conditions, determined by the instructions and the nature of the cues on their recall sheets. The order of lists was balanced between two groups of subjects in each condition. One group of subjects received no cues and were instructed to recall as many items as possible in any order (free recall, or FR). Another group of subjects was given the same instruction but was provided with the names of the eight list categories as cues ( $\mathrm{R}-8 \mathrm{C}$. for recall with eight category names). In the other two conditions, subjects were given four category names from the list. In one condition subjects were told to recall as many items as possible from the list, both items in the cued categories and as many items as possible from the other categories (the R-4C condition). In the other condition subjects were also given four category names, but they were told to recall only items from the four categories whose names were not provided. (Since these subjects could forget or ignore the cued categories, this condition is referred to as $\mathrm{F}-4 \mathrm{C}$, for forget four categories.)

Procedure. All subjects were given instructions at the beginning of the experiment explaining its general nature. They were informed of the categorized nature of the lists they were to remember and the number of categories in the lists, 
but the number of items in each category was not specified. They were simply told that they would be asked to recall as much of the list as possible later, after they had performed an interpolated task and read a brief instruction at the top of their recall sheets telling them how to recall the list. The subjects were asked to record their responses in columns and told that they would be asked to draw a line under the last word they had recalled every $90 \mathrm{sec}$ during the recall period.

Items in the list were presented via tape recorder at a $2-\mathrm{sec}$ rate and were blocked as to category, with items from each category preceded by the category name. Category names were presented in cadence with category instances but were distinguished by intonation. Following presentation of each list, all subjects were engaged in one of two interpolated tasks for $3 \mathrm{~min}$. The two tasks were recall of the presidents of the United States or recall of the 50 states.

After the interpolated task, subjects were told to turn to their recall sheets and read the instructions at the top. Instructions on the four different kinds of sheets were of roughly equal length. Free recall subjects and $\mathrm{R}-4 \mathrm{C}$ subjects were told to recall as many items as possible in any order. Subjects in the $\mathrm{R}-4 \mathrm{C}$ condition were given the following instructions: "Your task is to recall all the items you can from the list you just heard. However, please be certain to recall as many items as possible from the four categories at the top of the sheet. Remember, recall as many items as you can, but especially items from these four categories." Subjects in the $\mathrm{F}-4 \mathrm{C}$ condition were told to recall as many items as possible from the list "except for items in the four categories listed below. You may try to forget items from these categories and just concentrate on recalling other items from the list."

There were 24 subjects in each of the four conditions. Four categories were randomly selected from the pool of eight in each list for each subject in the $\mathrm{R}-4 \mathrm{C}$ and $\mathrm{F}-4 \mathrm{C}$ conditions. One subject in each of these conditions received the same four category names at the top of their respective recall sheets. In the R-8C condition, these four category names were listed prior to the other four. The recall period was $7.5 \mathrm{~min}$, with subjects instructed to draw a line under the last word they had written every $1.5 \mathrm{~min}$.

The cuing conditions were arranged so that subjects in all four conditions could be scored on equivalent sets of 32 critical items. Critical items were those that did not appear on the recall sheets of the $\mathrm{F}-4 \mathrm{C}$ and $\mathrm{R}-4 \mathrm{C}$ subjects. Thus, they were the only items that $\mathrm{F}-4 \mathrm{C}$ subjects recalled and were the items for which $R-4 C$ subjects did not receive category names. For $R-8 C$ subjects the four critical category names were written after those for the other four categories on the top of the recall sheets, and thus critical categories tended to be recalled after other categories. For free recall subjects, the critical items were simply a random half of the words. Thus, the four groups of subjects were yoked in terms of specific items to be recalled and this yoking was done on the basis of individual subjects; that is, the set of critical items differed for yoked groups of four subjects.

\section{Results and Discussion}

Presented in Figure 1 is the mean cumulative recall of the equivalent sets of critical items for the four conditions of Experiment 1. Recall of critical items was best in the R-8C condition at the end of the recall period (11.8 items recalled), and somewhat less in the free recall and F-4C conditions (10.8 and 9.4 words recalled). Recall of critical items in the $\mathrm{R}-4 \mathrm{C}$ condition was much poorer than in the other three conditions: Only 6.4 critical items were recalled. An analysis of variance indicated reliable variation among conditions $[\mathrm{F}(3,92)=8.31, \quad \mathrm{MSe}=16.00]^{1}$. Tests with the

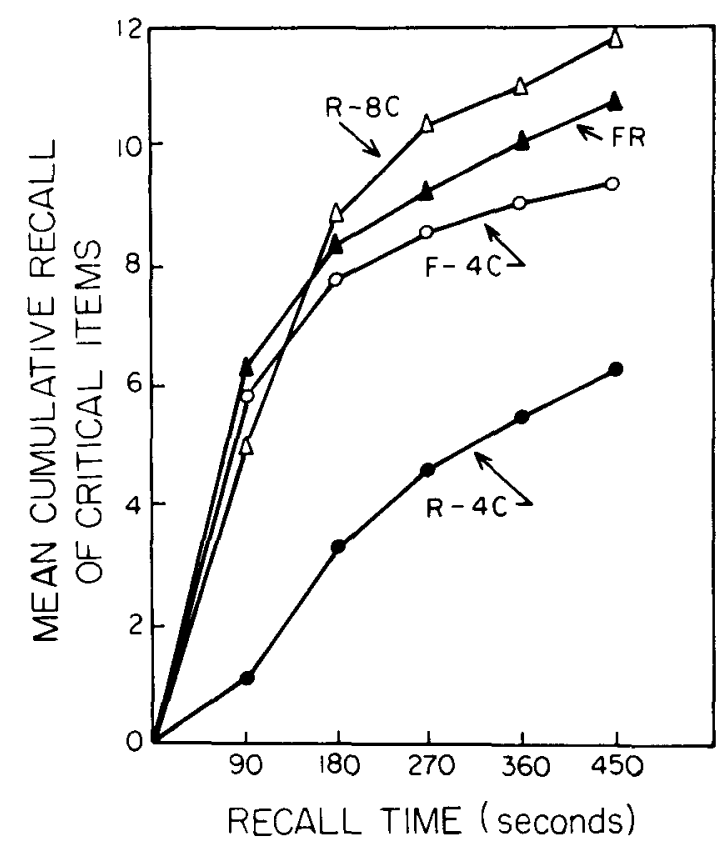

Figure 1. Mean cumulative recall of critical items in the four conditions of Experiment 1.

Newman-Keuls procedure indicated that only the difference between the R-4C condition and the other three conditions was reliable. It is not particularly surprising that R-8C subjects did not differ from free recall subjects in critical item recall, since in this condition the names of the four critical categories appeared after the other category names and the size of the cued recall advantage in Figure 1 is reduced by scoring only half the items. When scored on all items, R-8C subjects recalled 25.0 words ( 7.4 categories), while FR subjects recalled only 21.2 words (5.8 categories).

Presented in Table 1 is recall of critical items, categories, and items per category for the four conditions. Subjects were given credit for recall of a critical category when they had recalled at least one word from the category. It is apparent from Table 1 that the main difficulty subjects in the $\mathrm{R}-4 \mathrm{C}$ condition had in recalling critical items was in gaining access to the categories rather than recalling items within categories. Free recall subjects recalled words representing 3.0 of the 4 critical categories, while R-4C subjects recalled words from only 1.7 of the same categories. However, recall of items within categories

Table 1

Mean Recall of Words ( $R w$ ), Categories (Rc), and Words per Category $(\mathrm{Rw} / \mathrm{c})$ in the Four Conditions of Experiment 1

\begin{tabular}{cccc}
\hline Condition & $\mathrm{Rw}$ & $\mathrm{Rc}$ & $\mathrm{R} / \mathrm{wc}$ \\
\hline R-8C & 11.8 & 3.7 & 3.19 \\
FR & 10.8 & 3.0 & 3.60 \\
F-4C & 9.4 & 2.8 & 3.36 \\
R-4C & 6.4 & 1.7 & 3.76 \\
\hline
\end{tabular}


was about the same for these two conditions ( $3.60 \mathrm{vs.}$ 3.76). Thus the interference exerted from recall of the first four categories under $\mathrm{R} 4 \mathrm{C}$ conditions affected access to additional categories, not recall of items within categories.

It should be pointed out that although the R-4C subjects recalled fewer critical words and categories than did FR subjects, they recalled more cued words and categories. The R-4C subjects recalled 15.0 words representing 3.98 categories of those that were cued, while free recall subjects recalled 10.4 words representing 2.89 categories when scored on the same material. (The maximum possible is four categories and 32 words.) Thus cuing with only part of the category names from a list enhanced recall of the cued categories relative to free recall, but reduced recall of noncued categories. This reciprocal facilitation and impairment of recall has also been reported in other situations (e.g., Brown, 1968; Tulving \& Hastie, 1972).

The present result is quite similar to one reported by Parker and Warren (1974) and serves to clarify their result. They presented two groups of subjects with 40 words, 2 words from each of 20 categories. One group of subjects (the control group) was asked for free recall of the words after reading aloud 10 numbers, while the experimental subjects recalled the words after reading aloud 10 of the category names. The experimental subjects were further told "that their optimal strategy would be to first recall as many words as they could without using the index cards on which the category names were typed. After doing this they could try to use the index cards to facilitate further retrieval" (Parker \& Warren, 1974, p. 1,123). Thus it appears that Parker and Warren's (1974) experimental condition was quite similar in design to the F-4C condition in the present experiment, since they intended for subjects to recall words from noncued categories after exposure to names of other categories from which subjects were not to recall (at least initially). Parker and Warren found that recall of noncued words was reliably poorer in their experimental than control condition. However, it seems unlikely that their experimental subjects followed the direction to recall noncued words prior to cued words since even in the first $20 \%$ of the items recalled, recall of cued words was greater than noncued words (Parker \& Warren, 1974, Table 2). Thus, their experimental condition was probably more like the R-4C condition in Experiment 1 . From the results of the F-4C condition in the present experiment, it seems likely that there is little, if any, negative effect from simply having subjects read category names prior to recalling other categories. Recall of critical items was not reliably poorer in a statistical sense than that of free recall subjects scored on the same items. However, F-4C subjects did perform less well than free recall subjects in critical item recall on both lists, so there is some evidence for the experimental reliability of the effect. At any rate, a comparison of recall in the present experiment between the $\mathrm{F}-4 \mathrm{C}$ and $\mathrm{R}-4 \mathrm{C}$ conditions indicates that recall of critical items is much poorer after recall of words from the cued categories (R-4C) than after the category names have simply been presented (F-4C). Since subjects in Parker and Warren's (1974) study began recalling cued items early in the recall period, it is likely that the reliably poorer recall of noncued words in the experimental condition is similarly attributable to prior recall of cued categories.

Both the present results and those of Parker and Warren (1974) may have been affected by the amount of time spent in recall of cued and critical items. In the present experiment, subjects in the R-4C condition may simply have spent more time in recall of words from the cued categories to the relative neglect of recall of words in the critical categories. Free recall subjects would, of course, exhibit no such tendency, since for them the critical categories were simply a random half of the categories. The reason critical items were so poorly recalled by $\mathrm{R}-4 \mathrm{C}$ subjects, then, might have been simply that they spent most of their time recalling words from the cued categories. Since recall was measured cumulatively in the present experiment, information can be gained on whether or not subjects in the different conditions had reached asymptote in cumulative item recall. Examination of Figure 1 indicates that only in the F-4C condition did recall appear to be near asymptote. In the other three conditions, recall was still increasing even between 6 and $7.5 \mathrm{~min}$. Further, recall increased most rapidly in the $\mathrm{R}-4 \mathrm{C}$ condition. Although it seems unlikely, the possibility remains that subjects in this condition would have eventually recalled as many items as free recall subjects if given additional time.

Parker and Warren (1974) attempted to blunt the possibility of subjects' spending greater time recalling cued than noncued items in their experimental condition by asking subjects to recall the noncued items first. As already discussed, internal analyses indicate that this strategy was at best only partially successful, but since subjects were only allowed $2.5 \mathrm{~min}$ for recall in their experiment, it is unlikely that recall was near asymptote.

\section{EXPERIMENT 2}

The purpose of the second experiment was to replicate and extend the results of Experiment 1 and to eliminate the problem of limited recall time. In Experiment 2 subjects were presented only 50 words (10 categories with 5 words per category) and at the time of recall were given zero, two, four, or seven category names with instructions to recall as many items from the list as possible, both cued and noncued items. It was expected that recall of critical items would 
decrease with greater numbers of category names presented as cues. A 10-min recall period was employed and recall was measured cumulatively.

\section{Method}

The method was quite similar to that of Experiment 1. The 76 subjects were Purdue undergraduates serving in partial fulfillment of a course requirement. After general instructions, the subjects heard and recalled four categorized lists presented at a 2 -sec rate. Each list contained 10 categories with five items per category. The words were blocked as to category and preceded by the category name during presentation. The 40 categories and words were selected from the norms of Battig and Montague (1968), Hunt and Hodge (1971), and Shapiro and Palermo (1970).

Following presentation of each list, subjects turned a page in their answer booklets and were assigned to a particular condition by the instruction at the top of the page. The instruction in all four conditions stated, "You are to recall all the items you can from the list you just heard in any order you want." The four different conditions were defined by the number of category names subjects received at the top of their sheets, zero, two, four, or seven. Subjects who received category names were further instructed that "we want you to be especially certain to recall items from the following $[2,4$, or 7$]$ categories, but try your best to recall as many items as possible from all 10 categories." Free recall subjects also received a second sentence that simply reiterated that they should try to recall as many items as possible in order to equate the length of instructions for the four groups of subjects. Reading the instructions and, in three conditions, the category names after the presentation of the list was intended to eliminate information held in primary memory.

The experimental design was completely within subjects, so that each subject was assigned to one of the four experimental conditions after presentation of each list. The lists were presented in the same order to all subjects, but the particular conditions were systematically counterbalanced across subjects by a balanced Latin square for groups of four subjects. Thus the basic counterbalanced experimental design was replicated 19 times on groups of four subjects. Similarly, for each group of four subjects, three categories from each list were randomly selected to be critical categories. The R-7C subjects received the other seven as cues, the $\mathrm{R}-4 \mathrm{C}$ subjects received a random subset of these seven as cues, and the $R-2 C$ subjects received a random subset of the four categories of the $\mathrm{R}-4 \mathrm{C}$ subjects. Thus all four groups of subjects could be scored on a group of three critical categories or 15 critical words, the categories that the R-7C subjects did not receive for each list.

Subjects were allowed $10 \mathrm{~min}$ to recall each list and were asked to draw a line every minute after the last word they had written. Subjects were tested in groups of 4 to 12 . The experimental session lasted slightly over $1 \mathrm{~h}$.

\section{Results and Discussion}

The primary results of interest are cumulative recall of the 15 critical items, those items that were not cued with category names in any of the four conditions. These functions appear in Figure 2. Free recall subjects recalled most critical items by the end of the recall period, R-2C and $\mathrm{R}-4 \mathrm{C}$ subjects recalled roughly the same number of critical items, and R-7C subjects recalled fewest critical items. An analysis of variance indicated that there was reliable variation among conditions $[F(3,225)=9.35, \mathrm{MSe}=10.78]$. Newman Keuls tests revealed that there were reliable differences

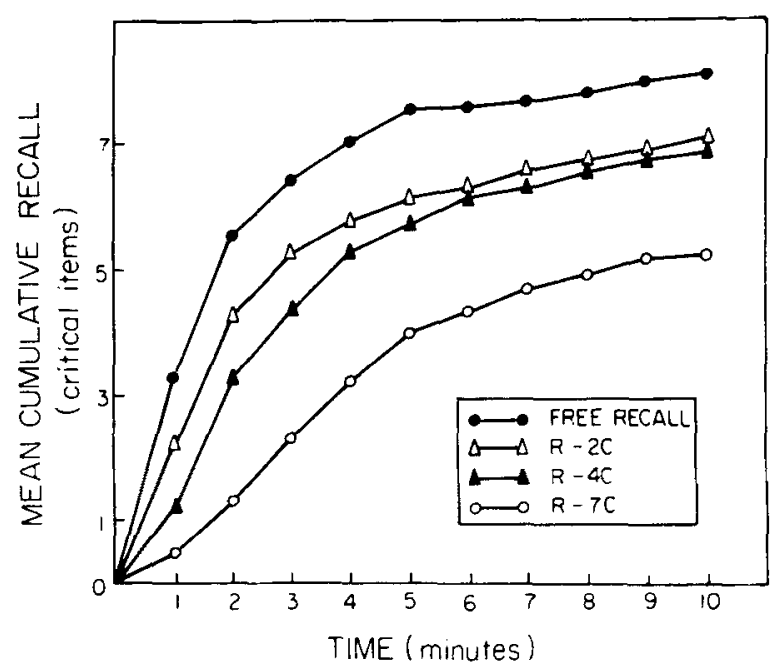

Figure 2, Mean cumulative recall of critical items in the four conditions of Experiment 2.

between free recall and the other three conditions and between $\mathrm{R}-7 \mathrm{C}$ and the other three conditions. The R-2C and R-4C conditions did not differ in terms of number of critical items recalled.

The results confirm the finding of Experiment 1 that presenting subjects with category names reduces recall of items from noncued categories. This cannot be attributed to a lack of time to recall noncued items in this experiment, since the cumulative recall curves appear to be at asymptote. In addition, these results show that there is, in general, a tendency for recall of noncued words to be reduced with increases in the number of category names presented. The only exception is the lack of difference between recall in the R-2C and R-4C conditions. Since subjects were told that they could recall items in any order, some subjects attempted to recall noncued categories before cued categories, which may have attenuated the effect. This tendency to recall noncued categories first was greatest in the R-2C condition and was exhibited less in the $\mathrm{R}-4 \mathrm{C}$ condition, but all subjects in the R-7C condition began recall with a cued category.

Presented in Figure 3 are cumulative recall curves of the three critical categories for the four conditions. Credit was given for recall of a category when the first word from that category was recalled. The pattern of results in Figure 3 closely resembles that of Figure 2, indicating that, as in the first experiment, the difficulty in recalling the critical items was in gaining access to the categories rather than to the items within categories. Recall of critical items within recalled categories was $3.58,3.54,3.45$, and 3.49 for the FR, R-2C, R-4C, and $\mathrm{R}-7 \mathrm{C}$ conditions, respectively.

Although presentation of only some of the category names reduced recall of noncued items relative to free recall, the beneficial effect of recall from the cued categories served to overcome the deficit produced by 


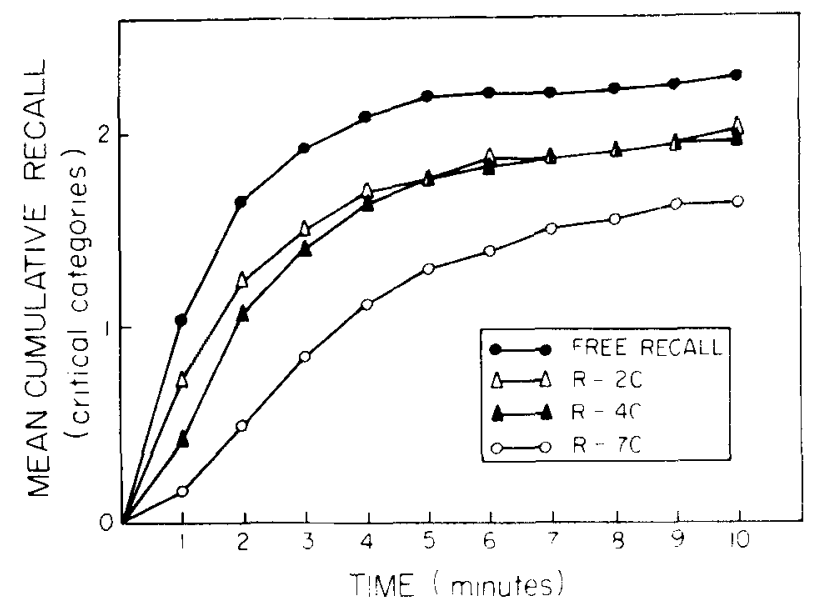

Figure 3. Mean cumulative recall of critical categories in the four conditions of Experiment 2.

recall interference in total item recall. Mean cumulative recall of all items and all categories is presented in the top and bottom panels, respectively, of Figure 4. There is little difference among the conditions, except that recall is somewhat better in the $\mathrm{R}-7 \mathrm{C}$ condition than the other three. The same pattern of results is evident, once again, in both recall of words and categories. Presenting category names aids recall of words in the cued categories relative to free recall, but depresses recall of noncued categories. These complementary effects roughly offset each other in the four conditions of the present experiment to produce little difference among conditions in overall levels of recall.

\section{EXPERIMENT 3}

The first two experiments showed that when subjects were given only some category names following presentation of a categorized list, recall of the other categories was impaired relative to free recall. Recall of cued categories interferes with recall of noncued categories. However, there is another possible interpretation of this phenomenon. Perhaps recall merely serves as a difficult interpolated task, much like counting backward by threes or other verbal interpolated tasks, and simply delays recall of critical items. Free recall subjects may recall critical items better simply because it is possible to recall them earlier during the recall period. The Parker and Warren (1974) study attempted to circumvent this problem by asking subjects to recall noncued categories before cued categories, but as previously noted, it is unclear as to how well subjects followed this instruction.

Experiment 3 was directed at obtaining more evidence on this issue. After listening to a long categorized list, subjects either received cues for recall of half of the items from the list or were distracted with several different types of interpolated task. Following this initial period, subjects were asked to recall all the remaining items from the list. If recall simply serves as an interpolated task delaying further recall, one would expect that the subjects who recalied the first half of the list during the initial period would recall the remaining critical items no worse than subjects performing the neutral interpolated tasks during the initial period. However, if recall interference is specific to the act of recall of the early categories from the list, then there should still be impairment from recalling the early categories relative to performing the other interpolated tasks during the initial period.

\section{Method}

Subjects. The subjects were 240 Purdue University undergraduates who served to fulfill part of a course requirement. They were tested in groups of 10 to 20 in experimental sessions that lasted about $30 \mathrm{~min}$.

Materials. Two lists were prepared and presented via tape recorder at a $2-\mathrm{sec}$ rate. One list was composed of 50 unrelated words, selected from the Paivio, Yuille, and Madigan (1968) norms, that varied widely in imagery and other dimensions. The second list contained 100 words, 5 words from each of 20 categories selected from the category norms mentioned previously.

Design and Procedure. There were five conditions in the experiment. The design was between subjects, with 48 subjects in each condition. At the beginning of the experiment, all subjects were told that they would be asked to listen to and recall in any order a list of common English words. Then the list of 50 unrelated words was presented at a $2-\sec$ rate and subjects were allowed $3 \mathrm{~min}$ to recall it. Next, subjects heard the categorized word list and then spent a 5 -min period engaged

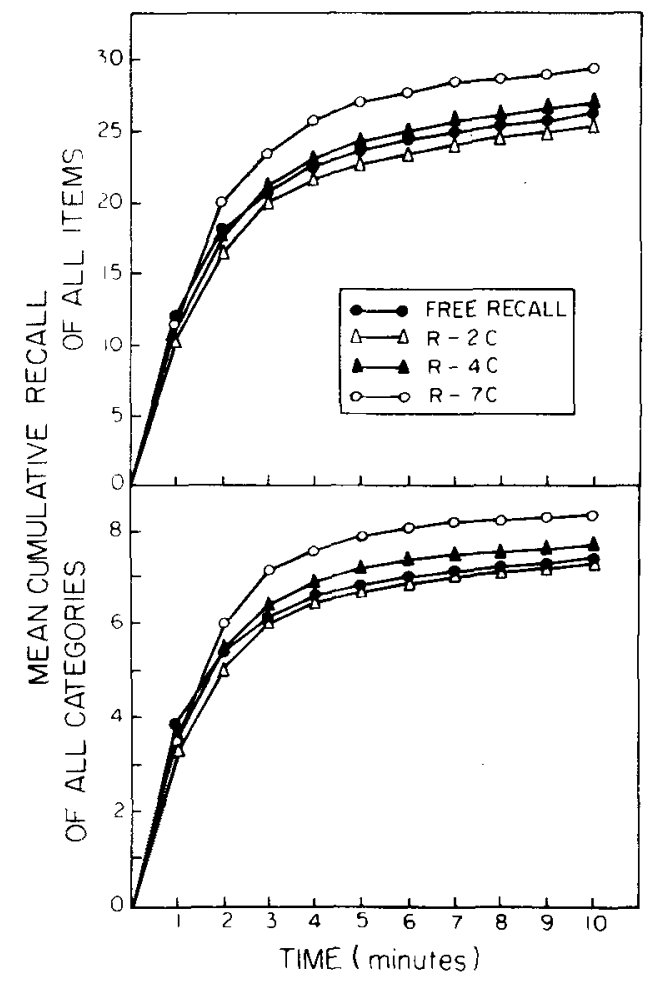

Figure 4. Mean cumulative recall of all words (top panel) and all categories (bottom panel) in the four conditions of Experiment 2. 
in various tasks. One group of subjects (the "read" condition) was given a brief passage on the "origins of modern science," by A.N. Whitehead, that pretesting had shown to take about $5 \mathrm{~min}$ to read. Subjects in the other four conditions were all asked to recall 50 items during the 5 -min period. Subjects in the "list" condition were asked again to recall as many items as possible from the unrelated word list. Subjects in the "states" condition were asked to recall as many of the 50 states as possible. Subjects in the other two conditions were given 10 category names from the list and were asked to recall as many words as possible from only these 10 categories. These two R-10C conditions were distinguished in the next phase of the experiment.

After the initial 5 -min period, all subjects were given a 10 -min recall period. Subjects in the read, list, and states conditions were instructed to recall as many words as possible from the categorized list in any order. They were further told that there were 20 categories in the list. Subjects in the R-10C conditions were told to recall only words from the 10 additional categories during the $10-\mathrm{min}$ recall period. The two groups were distinguished by whether or not they received category name cues for the 10 critical categories. The R-10C subjects who were not given cues (referred to as R-10C-FR) were told that they could look back at the previous sheet if they were uncertain as to whether or not a particular word or category had previously been recalled. The other condition is referred to as R-10C-CR for cued recall.

In summary, recall of the 50 critical items was deferred in all conditions by various interpolated tasks. In one case subjects were simply required to read a passage, while in the other four conditions they were to attempt to recall 50 items during the 5-min period. In two of the conditions, recall was either from semantic memory (recall of states) or episodic memory (recall of the first list), but was unrelated to material recalled from the categorized list. It is of interest to determine whether these three tasks (reading, recalling the states, recalling the first list) differ in their effect on later recall of the categorized list, but the primary interest is in whether or not they differ in their effect on critical item recall from that of the $\mathrm{R}-10 \mathrm{C}-\mathrm{FR}$ condition. If $\mathrm{R}-10 \mathrm{C}-\mathrm{FR}$ subjects recall fewer critical items than do subjects in the other three conditions, then the act of recalling the first 10 categories interferes with recall of critical items in a way that is specific and not due to some general interference effect.

\section{Results and Discussion}

The mean number of words recalled on the 50-word unrelated list was about equal for subjects in the five conditions, indicating that the groups were roughly equivalent in ability. The mean number of words recalled from the first list immediately after its presentation for the read, states, list, R-10C-FR, and R-10C-CR groups were $11.5,11.9,11.1,11.9$, and 12.3 words recalled, respectively.

Of primary interest is recall of critical words during the final $10-\mathrm{min}$ recall period. Interestingly, there were no reliable differences among the three control conditions. The subjects who read for $5 \mathrm{~min}$ recalled 19.8 critical words, while subjects who recalled the unrelated word list or the states of the U.S. recalled 19.2 and 19.3 critical words, respectively. (Recall of all 100 words for these three groups was $40.8,41.0$, and 39.4 mean words recalled, respectively). Thus attempting to recall 50 words from either semantic or episodic memory, from a category or list unrelated to the target list, did not impair recall of the categorized list relative to the condition where subjects simply read a short passage. The act of recalling these types of material did not produce any more general interference than did reading.

Since the read, list, and states conditions did not vary, results from these control conditions were combined for comparison with the R-10C-FR subjects in terms of the number of critical words recalled. The results are presented in Figure 5, where it can be seen that at the end of the recall period control subjects had recalled more critical words than had subjects who had previously recalled words from the 10 cued categories and were only responsible for the critical words during the final recall period (R-10C-FR subjects). The magnitude of the difference was not great (19.5 critical words recalled in the control condition and 17.7 words recalled in R-10C-FR condition), and in fact reached only a marginal level of statistical reliability $[t(190)=1.48, p=.08$, one-tailed $]$. However, as in the previous experiments, the difference between conditions was also reflected in terms of the number of categories recalled (6.0 in the control condition vs. 5.2 in the R-10C-FR condition). This difference did reach conventional levels of reliability $[\mathrm{t}(190)=2.44, \mathrm{p}<.01$, one-tailed]. Thus, category recall seems more sensitive a measure than number of words recalled. At any rate, there is evidence that the recall interference exhibited in the earlier experiments was not due simply to the general interfering effects of recall, but rather was specific to recall of categories from the list.

The magnitude of the recall interference effect was of modest size in the present experiment relative to

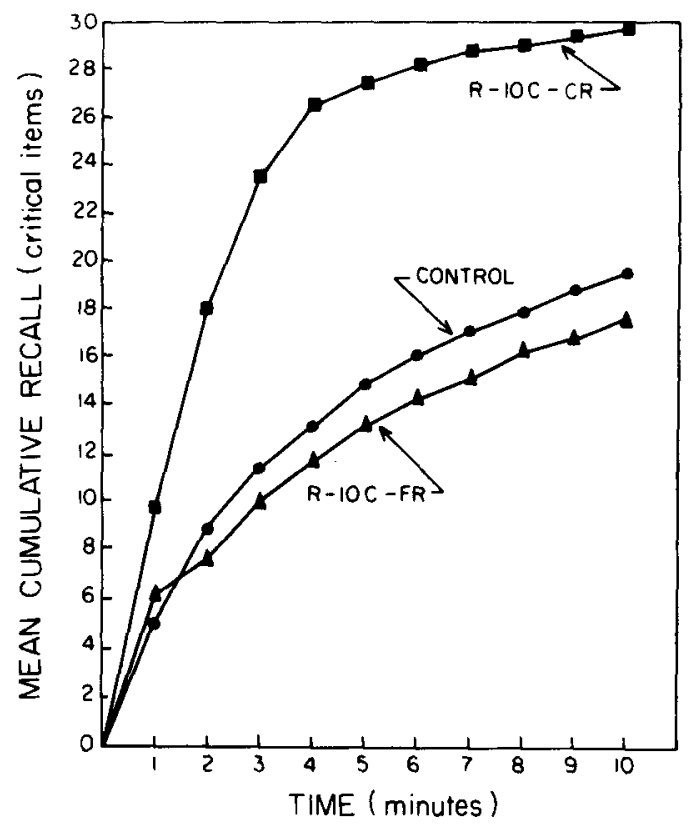

Figure 5. Mean cumulative recall of critical items in the conditions of Experiment 3. The control condition comprises the "read," "list," and "states" conditions, which did not differ from each other. 
Experiments 1 and 2. There are at least three potential reasons, though, that the observed difference between the R-10C-FR condition and the control conditions in Experiment 3 is a very conservative estimate of the amount of recall interference that actually occurred. Three factors operate against the discovery of large recall interference effects here. One factor is general across all three experiments, while the other two pertain only to Experiment 3 . In each experiment, there was presumably natural recall interference operating in the free recall condition. That is, free recall subjects do not recall the critical categories before the others, since the critical categories are merely a random half of the total set. Rather, recall of other categories may interfere with recall of critical categories in the free recall condition, as in the conditions where subjects received category names. It is merely the case that recall interference should be greater in cued recall than in free recall. The real effect of recall interference in limiting recall is undoubtedly underestimated by the free recall/cued recall comparisons in all of the present experiments.

In the third experiment, there was a second source of bias in that R-10C-FR subjects had twice as long to recall each of their target items as did free recall subjects. This is because free recall subjects were responsible for recalling all 100 words during the $10 \cdot \mathrm{min}$ recall period, while R-10C-FR subjects were only required to recall 50 (the critical words). The usual way to correct for a confounding of number of words to be recalled with recall time (for example, when list length is varied) is to allow subjects a constant amount of time per to-be-recalled word. If this convention had been followed in the present study, the difference between the control and R-10C-FR conditions in terms of the mean number of critical words recalled would be 19.8 vs. 13.2 , the number of critical words recalled by R-10C-FR subjects after $5 \mathrm{~min}$.

Experiment 3 was designed to eliminate the problem of subjects receiving some category names as cues recalling the noncued critical items after a longer retention interval than the free recall subjects. However, in Experiment 3 the situation was exactly reversed; since control subjects had to recall all 100 words while R-10C-FR subjects recalled only the 50 critical words, recall of the critical words was delayed for the control subjects. When these three factors are taken into account, it is apparent that the magnitude of the recall interference effects are underestimated in all the current experiments, but most seriously in the third.

A final point is worth mentioning with regard to Experiment 3. The superior recall of the R-10C-CR subjects in Figure 5 indicates that the effect of recall interference is largely to reduce the accessibility rather than the availability of information. When the category names of the critical items are presented, many more words can be recalled. The R-10C-CR subjects recalled a mean of 29.7 words representing 9.4 categories.

\section{GENERAL DISCUSSION}

Results from the three experiments reported here indicated that providing only some of the category names from a categorized list as retrieval cues enhanced recall of words from the cued categories relative to free recall, but reduced recall of the other categories from the list. The greater the number of category names presented, the greater was the decrement in recall of the noncued categories. The recall interference was specific to recall of categories and did not affect recall of words within recalled categories. The interference exerted by recall was also specific to previous recall of other categories from the list and was not simply due to general interference from the act of recall. In the third experiment, subjects who recalled either the 50 states or a 50 -item unrelated word list recalled the words in the categorized list no more poorly than did subjects who had been distracted by reading a short essay.

The present results and those of Parker and Warren (1974) appear to be inconsistent with the idea that higher order units (categories, in the present experiment) are recalled by direct associations from one to another. If additional categories were recalled by direct associations from previously recalled categories, one would expect presentation of some category names from the list to increase rather than diminish recall of the remaining categories. There is a direct parallel here to the part-list cuing experiments of Slamecka and others (Roediger, 1973; Rundus, 1973; Slamecka, 1969) where it was concluded that words are not directly interassociated since providing some words as cues failed to aid, and in fact hindered, recall of other words.

The fundamental problem that these experiments and others in this series (e.g., Roediger, 1973; Roediger, Stellon, \& Tulving, 1977) address is the relationship between the facilitating and inhibiting effects of recall and the similar effects produced by different types of retrieval cues. The results of the present experiments indicate that recall is in some ways a self-limiting process; cued recall of some categories reduced free recall of other categories from the same list relative to free recall of the entire list. On the other hand, there is ample evidence that in some ways recall is also a self-propagating process and that the provision of cues can often aid recall (e.g., Tulving \& Pearlstone, 1966). How are these offsetting facilitating and inhibiting effects of recall to be characterized?

In order to answer this question, it is useful to introduce the notion of two different types of association, which may be referred to as horizontal and vertical associations (Segal \& Mandler, 1967; Wickelgren, 1976). In the literature concerned with recall of categorized lists, category clustering has been explained as mediated by direct (horizontal) associations (Deese, 1959) or by associations from a higher level (vertical associations) to words within the categories 
(Bousfield \& Cohen, 1953). Both types of associations are employed in Anderson's (1972) FRAN model. Evidence from part-list cuing studies argues that the issue should be decided in favor of vertical or hierarchical associative theories with no need to postulate direct associations among category instances (Roediger, 1973; Slamecka, 1972).

This general viewpoint that only vertical associations are necessary to explain organized recall has been developed and extended by Rundus (1973), who based his work in large part on Shiffrin's (1970) theory of recall. Rundus' (1973) view of the retrieval process underlying recall rationalizes the complementary propagating and limiting aspects of recall and is useful in understanding the results of the experiments reported here. Briefly, it is assumed that information from categorized lists is stored in a hierarchical arrangement with individual elements (words) stored below a higher level control element that can be represented by the category name. The term control element (Estes, 1972) is used here to indicate that the recallability of the words is controlled by the higher order representation. The elements representing the categories are also associated to a higher order representation that may be thought of as a "list" presentation; that is, it indicates that the categories all occurred in the particular list most recently studied. The elements nested below each control element vary in terms of their strength of relationship to the control element and this determines order of recall. However, it is further assumed that the act of recalling an element increases the strength of its representation to its higher order control element much as would additional study time. The final crucial assumption is that the retrieval process underlying recall involves sampling with replacement such that when a word or category has been recalled, it is not eliminated from consideration on future retrieval attempts. In fact, because recalled information has been strengthened, it is more likely to be retrieved again to the exclusion of unrecalled information. The sampling-with-replacement nature of recall is probabilistic, but operates such that the more information that has been recalled, the less the likelihood that further information can be recalled. Eventually, recall is terminated when no new information is retrieved for a certain period of time or for a certain number of retrieval attempts, although there should remain some probability of information being retrieved with continued effort.

This scheme allows for recall to be both selfpropagating and self-limiting. Access to a control element within a hierarchy facilitates access to subordinate elements and inhibits access to coordinate elements. Thus in the experiments reported here, providing category names aided recall for words in the cued categories due to vertical associations, but reduced recall of other categories because control elements of the cued categories were strengthened so that they were repeatedly retrieved to the relative exclusion of control elements of noncued categories. Further, the more categories from the list that were cued and thereby strengthened, the less did subjects recall the noncued categories. Finally, as one would predict from Rundus' model, the inhibiting effect of presenting some category name cues on recall of other words in the list is specific to recall of additional categories, not words within categories. The same scheme accounts in a similar manner for the inhibiting effect of part-list cues. In fact, it was originally devised for that purpose, but it also serves as a more general theory of recall and is potentially applicable to many other situations.

\section{REFERENCES}

ANDERSON, J. R. FRAN: A simulation model of free recall. In G. H. Bower (Ed.), The psychology of learning and motivation (Vol. 5). New York: Academic Press, 1972.

Batrig, W. F., \& Montague, W. E. Category norms for verbal items in 56 categories: A replication and extension of the Connecticut category norms. Journal of Experimental Psychology, 1969, 80(3, Part 2).

Bousfield, W. A., \& CoHEN, B. H. The effects of reinforcement on the occurrence of clustering in the recall of randomly arranged associates. Journal of Psychology, 1953, 36, 67-81.

BRowN, J. Reciprocal facilitation and impairment in free recall. Psychonomic Science, 1968, 10, 41-42.

Crowder, R. G. Principles of learning and memory. Hillsdale, N.J: Lawrence Erlbaum, 1976.

DEESE, J. Influence of inter-item associative strength upon immediate free recall. Psychological Reports, 1959, 5, 305-312.

Dong, T. Cued partial recall of categorized words. Journal of Experimental Psychology, 1972, 93, 123-129.

Estes, W. K. An associative basis for coding and organization in memory. In A. W. Melton \& E. Martin (Eds.), Coding processes in human memory. Washington, D.C: Winston, 1972.

Hunt, K. P., \& Hodge, M. H. Category-item frequency and category name meaningfulness $\left(m^{\prime}\right)$ : Taxonomic norms for 84 categories. Psychonomic Monograph Supplements, $1971,4,97-121$.

Paivio, A., Yuille, J. C., \& Madigan, S. Concreteness, imagery, and meaningfulness values for 925 concrete nouns. Journal of Experimental Psychology Monograph Supplement, 1968, 76(1, Part 2).

Parker, R. E., \& Warren, L. Partial category cueing: The accessibility of categories. Joumal of Experimental Psychology, 1974, 102, 1123-1125.

RoEDIGER, H. L. Inhibition in recall from cueing with recall targets. Journal of Verbal Learning and Verbal Behavior, $1973,12,644.657$.

Roediger, H. L. Inhibiting effects of recall. Memory \& Cognition, 1974, 2, 261-269.

Roediger, H. L., Stellon, C., \& Tulving, E. Inhibition from part-list cues and rate of recall. Journal of Experimental Psychology: Human Leaming and Memory, 1977, 3, 174-188.

Rundus, D. Negative effects of using list items as recall cues. Journal of Verbal Learning and Verbal Behavior, 1973, $12,43-50$.

Segal, M. A., \& Mandler, G. Directionality and organizational processes in paired-associate learning. Journal of Experimental Psychology, 1967, 74, 305-312. 
Shapiro. S. 1., \& Palermo, D. S. Conceptual organization and class membership: Normative data for representatives of 100 categories. Psychonomic Monograph Supplements. 1970. $3,107-127$.

Shiffrin, R. M. Memory search. In D. A. Norman (Ed.), Models of human memory. New York: Academic Press, 1970. Pp. 375-447.

Slamecka, N. J. An examination of trace storage in free recall. Journal of Experimental Psychology. 1968, 76, 504-513.

SLAMECKA, N. J. Testing for associative storage in multitrial free recall. Joumal of Experimental Psychology. 1969, 81, $557-560$.

Slamecka, N. I. The question of associative growth in the learning of categorized materials. Journal of Verbal Learning and Verbal Behavior, 1972, 11, 324-332.

Sмгтн, A. D. Output interference and organized recall from long-term memory. Journal of Verbal Learning and Verbal Behavior, 1971, 10, 400-408.

Smith, A. D., D'Agostino, P. R., \& Reid. L. Output interference in long-term memory. Canadian Journal of Psychology, 1970, 24, 85-87.

Tulving, E., \& Arbuckie, T. Y. Sources of intratrial interference in paired-associate learning. Journal of Verbal Learning and Verbal Behavior, 1963, 1, 321-334.
Tulving, E., \& Hastie, R. Inhibition effects of intralist repetition in free recall. Journal of Experimental Psychology, 1972. 92. 297-304.

Tulving, E., \& Pearlstone, Z. Availability versus accessibility of information in memory for words. Journal of Verbal Learning and Verbal Behavior, 1966, 5, 381-391.

WATKINS, M. J. Inhibition in recall with extralist "cues." Journal of Verbal Learning and Verbal Behavior, 1975, 14, 294-303.

WICKELGREN, W. A. Network strength theory of storage and retrieval dynamics. Psychological Review, 1976, 83, $466-478$

\section{NOTE}

1. Unless otherwise noted, all reliable effects involve $p$ values of less than .01 .

(Received for publication August 22, 1977; accepted October 4, 1977.) 